\title{
Cosmic censorship for some spatially homogeneous cosmological models.
}

\author{
Alan D. Rendall * \\ Max-Planck-Institut für Astrophysik $\dagger$ \\ Karl-Schwarzschild-Str. 1 \\ 8046 Garching bei München \\ Germany \\ and \\ Physics Department \\ Syracuse University \\ Syracuse NY 13244-1130 \\ USA
}

\begin{abstract}
The global properties of spatially homogeneous cosmological models with collisionless matter are studied. It is shown that as long as the mean curvature of the hypersurfaces of homogeneity remains finite no singularity can occur in finite proper time as measured by observers whose worldlines are orthogonal to these hypersurfaces. Strong cosmic censorship is then proved for the Bianchi I, Bianchi IX and Kantowski-Sachs symmetry classes.
\end{abstract}

\footnotetext{
* Supported in part by the NSF grant PHY90-16733 and research funds provided by Syracuse University

$\dagger$ Present address
} 


\section{Introduction}

The strong cosmic censorship hypothesis says that the maximal Cauchy development of generic initial data for the Einstein equations should be inextendible. Since the maximal Cauchy development is the largest region of spacetime which is uniquely determined by initial data, this is essentially the statement that the time evolution of a spacetime can generically be fixed by giving initial data. The question of the validity of strong cosmic censorship is thus the question of predictability in general relativity. The above definition is in fact only one partial formalisation of the general idea of strong cosmic censorship and is based on the suggestion of Eardley and Moncrief[5] that the right way of looking at the problem is as a question about global existence of solutions of the Einstein equations. That this is so will be taken for granted in what follows.

In any study of cosmic censorship where solutions of the Einstein equations are specified by giving initial data the choice of matter model is very important. The opinion has been expressed in the past that it is best to restrict to vacuum spacetimes, at least in the beginning. If cosmological spacetimes are considered then the direct physical relevance of vacuum models (representing a completely empty universe) is questionable. Thus from the point of view of physics the best justification for studying vacuum cosmological models seems to be the idea that what is learned in the process may usefully be applied at some later date to non-vacuum spacetimes. In the situations considered in the following the vacuum solutions appear to play an exceptional role and so some caution is required when treating vacuum cosmological models as indicators of the behaviour to be expected in cases when matter is present. At this time there are unfortunately no results available on the global structure of cosmological spacetimes containing matter comparable in generality to those which have been obtained in the vacuum case[3,4,8,13].

As soon as non-vacuum spacetimes are considered, certain problems arise with cosmic censorship. Phenomena like shell-crossing and shocks lead to singularities (or at least to situations which in our present state of mathematical sophistication we are forced to consider as singularities in rigorous analytical investigations) which intuitively are due purely to the matter itself and have little to do with gravitation. These may obscure the gravitational singularities which are the true object of interest in the study of cosmic censorship. These difficulties have been discussed in detail in [20] where evidence is presented that a good candidate for a matter model which avoids these problems is the collisionless gas, with the dynamics of matter being described by the Vlasov equation. In the asymptotically flat case a number of things have been proved about the properties of solutions of the Einstein equations coupled to matter of this type [14-17,19,21]. In the cosmological case much less is known although a general theorem concerning the long-time behaviour of a self-gravitating collisionless gas has been proved in the context of Newtonian cosmology[18]. The aim of this paper is to begin investigating the global dynamics of cosmological solutions of the Vlasov-Einstein system.

The simplest cosmological models are those which are spatially homogeneous and it is natural to look at these first. They fall into two broad classes, the Bianchi and Kantowski-Sachs models. The former can further be subdivided into Bianchi types IIX. (For general information on this see [23].) There is a huge literature concerning the dynamics of homogeneous cosmological models which generally relies on one of the following 
three methods:

a) finding explicit solutions

b) analysing a dynamical system in a small number of variables

c) replacing the full equations by some approximate ones.

The present work aims to obtain rigorous results concerning general solutions in a given symmetry class and so methods a) and c) are ruled out. Furthermore, even in the spatially homogeneous case the Vlasov-Einstein system involves infinitely many degrees of freedom (a function of the momentum variables) and so b) is also not an option here. A direct approach is necessary. The general idea is to first obtain the maximal Cauchy development by proving long-time existence in a constant mean curvature (CMC) slicing; in the homogeneous case the CMC slices coincide with the hypersurfaces of homogeneity. Once this has been done inextendibility can be checked by determining whether incomplete causal geodesics run into a curvature singularity.

The paper is organised as follows. Section 2 contains some generalities on Bianchi models and a proof that the occurrence of singularities in solutions of the Vlasov-Einstein system with a symmetry of this kind is controlled by the mean curvature of the hypersurfaces of homogeneity. A corresponding result for solutions with Kantowski-Sachs symmetry is obtained in section 3. These two sections also provide information on the qualitative behaviour of the mean curvature for the various symmetry classes. In section 4 it is shown that except in vacuum any singularity in a solution of the Vlasov-Einstein system with Kantowski-Sachs or Bianchi IX symmetry must be a curvature singularity, thus proving strong cosmic censorship in these two cases. It is also shown that the initial singularity is a curvature singularity for the other Bianchi types of Class A, once again under the restriction that the solution not be vacuum. To prove cosmic censorship for the remaining Bianchi types of Class A it would be sufficient to prove the completeness of future-directed causal geodesics. This is difficult and is carried out only for the simplest case, Bianchi type I, in the last section.

\section{Bianchi models}

Bianchi models are studied in this paper as a first step towards understanding more general cosmological solutions of the Vlasov-Einstein system and so it is appropriate to address the general question of which solutions of the Einstein equations should be regarded as cosmological. A conservative definition, and the one which will be adopted provisionally here, is that a cosmological spacetime is one which admits a compact partial Cauchy hypersurface. With this definition, the only Bianchi types which can occur for a spatially homogeneous cosmological model are I and IX. However this does not mean that the other Bianchi types are irrelevant in the present context because of the existence of locally spatially homogeneous cosmologies.

Definition A spacetime is called locally spatially homogeneous if each point has a neighbourhood which is isometric to an open subset of a spatially homogeneous spacetime.

With this definition, most Bianchi types can be realised in locally spatially homogeneous spacetimes possessing a compact Cauchy hypersurface. Discussions of which Bianchi types these are can be found in [1] and [6]. It can be shown that the universal cover of 
a locally spatially homogeneous spacetime is spatially homogeneous and since passing to the universal cover does not affect the dynamics it is enough to study the dynamics in the simply connected case. Then for Bianchi models the spatial manifold is in a natural way a simply connected 3-dimensional Lie group. The Kantowski-Sachs case, where passing to the universal cover brings no special advantage, is considered separately.

Let $G$ be a simply connected 3-dimensional Lie group and $\left\{e_{i}\right\}$ a left invariant frame on $G$. Denote the dual coframe by $\left\{e^{i}\right\}$. Consider a spacetime with underlying manifold $G \times I, I$ an open interval, and a metric of the form $-d t^{2}+g_{i j}(t) e^{i} \otimes e^{j}$. The initial value problem for the Vlasov-Einstein system will now be investigated in the case that the metric has this special form and the distribution function depends only on $t$ and $v^{i}$, where $v^{i}$ denotes the spatial components of the momentum in the frame $\left\{e_{i}\right\}$. Initial data will be given on the hypersurface $G \times\{0\}$. The constraints are

$$
\begin{aligned}
R-k^{i j} k_{i j}+\left(g^{i j} k_{i j}\right)^{2} & =16 \pi T_{00}, \\
\nabla^{i} k_{i j}-\nabla_{j}\left(g^{l m} k_{l m}\right) & =-8 \pi T_{0 j} .
\end{aligned}
$$

The evolution equations are

$$
\begin{aligned}
& \partial_{t} g_{i j}=-2 k_{i j} \\
& \partial_{t} k_{i j}=R_{i j}+\left(g^{l m} k_{l m}\right) k_{i j}-2 k_{i l} k_{j}^{l}-8 \pi T_{i j}-4 \pi T_{00} g_{i j}+4 \pi\left(g^{l m} T_{l m}\right) g_{i j} .
\end{aligned}
$$

These equations are written using frame components. The second fundamental form is denoted by $k_{i j} . R$ and $R_{i j}$ are the Ricci scalar and Ricci tensor of the three-dimensional metric respectively. The components of the energy-momentum tensor are denoted by $T_{00}$, $T_{0 i}$ and $T_{i j}$. The explicit form of the latter in terms of the phase space density $f$ of particles is

$$
\begin{aligned}
& T_{00}(t)=\int f\left(t, v^{k}\right)\left(1+g_{r s} v^{r} v^{s}\right)^{1 / 2}(\operatorname{det} g)^{1 / 2} d v^{1} d v^{2} d v^{3} \\
& T_{0 i}(t)=\int f\left(t, v^{k}\right) v_{i}(\operatorname{det} g)^{1 / 2} d v^{1} d v^{2} d v^{3} \\
& T_{i j}(t)=\int f\left(t, v^{k}\right) v_{i} v_{j}\left(1+g_{r s} v^{r} v^{s}\right)^{-1 / 2}(\operatorname{det} g)^{1 / 2} d v^{1} d v^{2} d v^{3}
\end{aligned}
$$

The Vlasov equation has the following form.

$$
\partial f / \partial t+\left[2 k_{j}^{i} v^{j}-\left(1+g_{r s} v^{r} v^{s}\right)^{-1 / 2} \gamma_{m n}^{i} v^{m} v^{n}\right] \partial f / \partial v^{i}=0
$$

The Ricci rotation coefficients $\gamma_{j k}^{i}$ are defined in terms of the structure constants $C_{j k}^{i}$ of the Lie algebra of $G$ by the following relations.

$$
\begin{aligned}
\gamma_{j k}^{i} & =\frac{1}{2} g^{i l}\left(C_{l j k}+C_{j l k}+C_{k l j}\right), \\
C_{i j k} & =g_{k l} C_{i j}^{l} .
\end{aligned}
$$


To have a complete set of equations it is necessary to compute $R_{i j}$ in terms of $g_{i j}$ but for the moment it is enough to know that $R_{i j}$ is of the form $(\operatorname{det} g)^{-n} \times\left(\right.$ polynomial in $g_{i j}$ and $C_{j k}^{i}$ ).

A local existence theorem will now be proved under the assumption that initial data $\left(g_{i j}^{0}, k_{i j}^{0}, f^{0}\right)$ are given with $f^{0}(v)$ a $C^{1}$ function of compact support. The characteristics of $(2.8)$ are the solutions $V^{i}(s, t, v)$ of the equation

$$
d V^{i} / d s=2 k_{j}^{i} V^{j}-\left(1+g_{r s} V^{r} V^{s}\right)^{-1 / 2} \gamma_{m n}^{i} V^{m} V^{n}
$$

with $V^{i}\left(t, t, v^{i}\right)=v^{i}$. To control the determinant of the metric the following consequence of (2.3) will be used.

$$
d / d t(\log \operatorname{det} g)=-2 g^{i j} k_{i j} .
$$

The mean curvature $g^{i j} k_{i j}$ will be of particular importance in the following and will be denoted from now on by $H$. Now define an iteration as follows. Let $f_{0}(t, v)=f^{0}(v)$, $g_{0}(t)=g^{0}$ and $k_{0}(t)=k^{0}$. If $f_{n}, g_{n}$ and $k_{n}$ are given for some $n$ determine $V_{n+1}$ by solving the characteristic equation (2.11) with $g$ and $k$ replaced by $g_{n}$ and $k_{n}$. Let

$$
f_{n+1}(t, v)=f^{0}\left(V_{n+1}(0, t, v)\right) .
$$

Next define an energy-momentum tensor $T_{n+1, \alpha \beta}$ by replacing $f$ by $f_{n+1}$ and $g$ by $g_{n}$ in (2.5)-(2.7). Now determine $g_{n+1}$ and $k_{n+1}$ by solving the linear ordinary differential equations which result when $T_{\alpha \beta}, g$ and $k$ are replaced by $T_{n+1, \alpha \beta}, g_{n}$ and $k_{n}$ respectively in the right hand side of (2.3) and (2.4) and $g$ and $k$ by $g_{n+1}$ and $k_{n+1}$ on the left hand side. Let $\left[0, T_{n+1}\right)$ be the maximal interval on which $g_{n+1}$ is positive definite. By induction it can be shown that $f_{n}, g_{n}$ and $k_{n}$ are $C^{1}$ on their domains of definition.

Let $|g|$ be the maximum modulus of any component $g_{i j}$ with a similar definition for $k_{i j}$. Now suppose that for all $n \leq N-1$ the following bounds hold.

$$
\left.\begin{array}{rl}
\left|g_{n}-g^{0}\right| & \leq A_{1} \\
\left(\operatorname{det} g_{n}\right)^{-1} & \leq A_{2} \\
\left|k_{n}-k^{0}\right| & \leq A_{3}
\end{array}\right\}
$$

Suppose further that $|v| \leq A_{4}$ whenever $f_{n}(t, v) \neq 0$. Here $A_{1}-A_{4}$ are positive constants which are for the moment arbitrary. The characteristic system (2.11) implies a bound of the form

$$
|v| \leq P_{0}+B_{4} t \text { whenever } f_{N}(t, v) \neq 0 .
$$

where $B_{4}$ depends only on $A_{1}-A_{4}$. As a consequence (2.5)-(2.7) imply a bound for $T_{N, \alpha \beta}$ depending only on $A_{1}-A_{4}$. Next (2.3) and (2.4) imply bounds of the form

$$
\left.\begin{array}{l}
\left|g_{N}-g^{0}\right| \leq B_{1} t \\
\left|k_{N}-k^{0}\right| \leq B_{3} t
\end{array}\right\}
$$


where $B_{1}$ and $B_{3}$ depend only on $A_{1}-A_{4}$. If $A_{1}-A_{4}$ (and hence $B_{1}$ ) are fixed then the inequalities (2.14) imply an inequality of the form

$$
\left(\operatorname{det} g_{N}\right)^{-1} \leq B_{2}
$$

whenever $t \leq T$ and $T$ is some positive time depending only on $A_{1}-A_{4}$. Now fix $A_{1}-A_{4}$ in such a way that $A_{2}>\left(\operatorname{detg}^{0}\right)^{-1}$ and $A_{4}>P_{0}$. Next reduce the size of $T$ if necessary so that $B_{1} T<A_{1}, B_{2}<A_{2}, B_{3} T<A_{3}$ and $P_{0}+B_{4} T<A_{4}$. Then it follows that all iterates exist on the interval $[0, T)$ and that $g_{n}$ and $k_{n}$ are bounded on that interval independently of $n$.

Consider next the difference of successive iterates for $n \geq 2$.

$$
\begin{aligned}
& \left|g_{n+1}(t)-g_{n}(t)\right|+\left|k_{n+1}(t)-k_{n}(t)\right| \\
& \leq C \int_{0}^{t}\left|g_{n}(s)-g_{n-1}(s)\right|+\left|k_{n}(s)-k_{n-1}(s)\right|+\left\|f_{n+1}(s)-f_{n}(s)\right\|_{\infty} d s .
\end{aligned}
$$

For the difference of the characteristics there is the estimate

$$
\left|d V_{n+1} / d s-d V_{n} / d s\right| \leq C\left(\left|V_{n+1}-V_{n}\right|+\left|g_{n}-g_{n-1}\right|+\left|k_{n}-k_{n-1}\right|\right)
$$

Let

$$
\begin{gathered}
\alpha_{n}(t)=\sup \left\{\left|V_{n+1}-V_{n}\right|(s, t, v): 0 \leq s \leq t, v \in \operatorname{supp} f_{n+1}(t) \cup \operatorname{supp} f_{n}(t)\right\} \\
+\left|g_{n+1}(t)-g_{n}(t)\right|+\left|k_{n+1}(t)-k_{n}(t)\right|
\end{gathered}
$$

Then

$$
\left\|f_{n+1}(t)-f_{n}(t)\right\|_{\infty} \leq\left\|f^{0}\right\|_{C^{1}} \alpha_{n}(t) .
$$

Inequalities (2.19)-(2.21) imply that

$$
\alpha_{n}(t) \leq C \int_{0}^{t} \alpha_{n}(s)+\alpha_{n-1}(s) d s
$$

Applying Gronwall's inequality to this gives

$$
\alpha_{n}(t) \leq C \int_{0}^{t} \alpha_{n-1}(s) d s
$$

It follows from this that $\alpha_{n}(t) \leq C^{n-2}\left\|\alpha_{2}\right\|_{\infty} t^{n-2} /(n-2)$ ! so that $\left\{g_{n}\right\},\left\{k_{n}\right\}$ and $\left\{V_{n}\right\}$ are Cauchy sequences on the time interval $[0, T)$. Denote the limits of these sequences by $g, k$ and $V$ respectively. Going back to (2.3) and (2.4), we see that $\partial_{t} g_{n}$ and $\partial_{t} k_{n}$ are uniformly convergent. Thus $f(t, v)=f^{0}(V(0, t, v))$ is $C^{1}$ and $(g, k, f)$ is a classical solution of equations (2.3)-(2.5) on the interval $[0, T)$. If two solutions with the same initial data are given define a quantity $\alpha(t)$ in terms of their difference in the same way that $\alpha_{n}(t)$ was defined in terms of the difference of two iterates. Going through the same steps as above leads to an estimate of the form $\alpha(t) \leq C \int_{0}^{t} \alpha(s) d s$. By Gronwall's inequality this means 
that $\alpha(t)$ is zero and hence that the two solutions agree. Thus the solution which has been constructed is uniquely determined by the initial data. If the initial data satisfy (2.1) and (2.2) then so does the solution of (2.3)-(2.5). To show this define

$$
\begin{aligned}
C & =R-k^{i j} k_{i j}+\left(g^{i j} k_{i j}\right)^{2}-16 \pi T_{00} \\
C_{i} & =\nabla^{j} k_{i j}+8 \pi T_{0 i}
\end{aligned}
$$

and note that (2.3)-(2.5) imply homogeneous first order ordinary differential equations for $C$ and $C_{i}$.

A close examination of the above proof shows that the size of $T$ is only restricted by the quantities:

$$
\left|g^{0}\right|,\left(\operatorname{det} g^{0}\right)^{-1},\left|k^{0}\right|,\left\|f^{0}\right\|_{\infty}
$$

and the diameter of $\operatorname{supp} f^{0}$. Thus if on some time interval $\left[0, T_{1}\right)$ the quantities $|g|$, $(\operatorname{det} g)^{-1},|k|,\|f\|_{\infty}$ and $\operatorname{diam} \operatorname{supp} f$ are bounded a solution exists on $[t, t+\epsilon)$ for any $t \in\left[0, T_{1}\right)$ and some $\epsilon$ independent of $t$. It can be concluded that the original solution can be extended to the larger interval $\left[0, T_{1}+\epsilon\right)$. The following result has now been proved.

Lemma 2.1 Let $\left(g^{0}, k^{0}, f^{0}\right)$ be an initial data set for equations (2.3), (2.4) and (2.8) which has Bianchi symmetry, satisfies the constraints (2.1) and (2.2) and is such that $f^{0}(v)$ is a $C^{1}$ function of compact support. Then there exists a unique corresponding maximal $C^{1}$ solution $(g, k, f)$ on an interval $\left[0, T^{*}\right)$. If $|g|,(\operatorname{det} g)^{-1},|k|$ and $\operatorname{diam} \operatorname{supp} f$ are bounded on $\left[0, T^{*}\right)$ then $T^{*}=\infty$.

In order to investigate the question of global existence the mean curvature will be examined.

$$
\partial_{t} H=R+H^{2}-12 \pi T_{00}+4 \pi g^{i j} T_{i j}
$$

This equation can usefully be combined with the Hamiltonian constraint (2.1). An equation which can be obtained in this way is

$$
\partial_{t} H=k^{i j} k_{i j}+4 \pi\left(T_{00}+g^{i j} T_{i j}\right)
$$

which shows that $H$ is non-decreasing. Now suppose that $H$ is bounded on $\left[0, T^{*}\right)$. Then from (2.28)

$$
H(t) \geq H(0)+\int_{0}^{t}\left(k^{i j} k_{i j}\right)\left(t^{\prime}\right) d t^{\prime}
$$

Thus $\int_{0}^{T^{*}}\left(k^{i j} k_{i j}\right)(t) d t<\infty$.

At this point a short interlude on linear algebra is necessary. Let $A$ and $B$ be $n \times n$ symmetric matrices with $A$ positive definite. For any matrix $C$ the norm is defined as usual by

$$
\|C\|=\sup \{\|C x\| /\|x\|: x \neq 0\} .
$$

It is also possible to define a relative norm by

$$
\|B\|_{A}=\sup \{\|B x\| /\|A x\|: x \neq 0\} .
$$


It follows immediately that $\|B\| \leq\|B\|_{A}\|A\|$. The other fact which is needed here is that

$$
\|B\|_{A} \leq \sqrt{\operatorname{tr}\left(A^{-1} B A^{-1} B\right)} .
$$

This can be proved by noting that there exists a basis $\left\{z_{1}, z_{2}, z_{3}\right\}$ with the property that $B z_{i}=\lambda_{i} A z_{i}$ for each $i$. The left hand side of (2.32) is then given by the maximum modulus of any $\lambda_{i}$. On the other hand, the eigenvalues of $A^{-1} B A^{-1} B$ are $\left\{\lambda_{i}^{2}\right\}$ so that the right hand side of $(2.32)$ is equal to $\left(\sum_{i} \lambda_{i}^{2}\right)^{1 / 2}$. Let $\|g\|$ and $\|k\|$ denote the norms of the matrices with entries $g_{i j}$ and $k_{i j}$ respectively. Let $\|k\|_{g}$ be the relative norm of the matrix with entries $k_{i j}$ with respect to the matrix with entries $g_{i j}$. Then

$$
\begin{aligned}
\|g(t)\| & \leq\|g(0)\|+2 \int_{0}^{t}\|k(s)\| d s \\
& \leq\|g(0)\|+2 \int_{0}^{t}\|k(s)\|_{g(s)}\|g(s)\| d s \quad . \\
& \leq\|g(0)\|+2 \int_{0}^{t}\left(k^{i j} k_{i j}\right)^{1 / 2}(s)\|g(s)\| d s
\end{aligned}
$$

Applying Gronwall's lemma to (2.33) gives

$$
\|g(t)\| \leq\|g(0)\| \exp \left[2 \int_{0}^{t}\left(k^{i j} k_{i j}\right)^{1 / 2}(s) d s\right] .
$$

By what has been said above it can be concluded that if $T^{*}$ is finite then $|g|$ is bounded on $\left[0, T^{*}\right)$. Equation $(2.12)$ shows that $(\operatorname{det} g)^{-1}$ is bounded on that interval. It is known that if $\operatorname{det} g$ and its inverse are bounded the scalar curvature $R$ is bounded from above[9]. Thus, from (2.1), $k^{i j} k_{i j}$ is bounded on $\left[0, T^{*}\right)$. Using the inequality $\|k(s)\| \leq\left(k^{i j} k_{i j}\right)^{1 / 2}\|g(s)\|$ we see that $|k|$ is bounded. The boundedness of $|g|$ and $(\operatorname{det} g)^{-1}$ implies that $g$ is uniformly positive definite on the interval of interest. Thus the solutions of the characteristic equation are bounded there. These statements add up to a stronger version of Lemma 2.1.

Lemma 2.2 Suppose that the hypotheses of Lemma 2.1 hold. If $H$ is bounded on $\left[0, T^{*}\right)$ then $T^{*}=\infty$.

Lemma 2.2 shows that the long time behaviour of $H$ gives useful information about the long time behaviour of the solution. Assume now without loss of generality that $H \leq 0$ for $t=0$. If this is not true for a given solution it may be arranged by doing the transformation $t \mapsto-t$. For all Bianchi types other than IX the scalar curvature $R$ is non-positive[9]. Hence equation (2.1) implies that for those types $H$ cannot become zero except in very special circumstances. More specifically, $H=0$ implies that $T_{00}=0$ (so that the solution is vacuum), $k_{i j}=0$ and $R=0$. Reference to [9] shows that for these Bianchi types the only cases where $R=0$ is possible are types $\mathrm{I}$ and $\mathrm{VII}_{0}$. Moreover the metrics for which this condition is satisfied are flat. Thus the spacetime must be Minkowski space. To sum up: if the Bianchi type is not IX and the spacetime is not flat then $H<0$ for all 
time. Lemma 2.2 then implies that $T^{*}=\infty$. Next note that $k^{i j} k_{i j} \geq 1 / 3\left(g^{i j} k_{i j}\right)^{2}$ so that (2.28) gives the inequality

$$
\partial_{t} H \geq \frac{1}{3} H^{2}
$$

Comparing the solution of this equation with the solution of $\dot{u}=1 / 3 u^{2}$ with the same initial value shows that if $H$ ever becomes positive then it diverges to $+\infty$ in finite time. Thus if it were known that $H$ always became positive in type IX the ultimate fate of $H$ would be known in all cases. That this is the case has been shown by Lin and Wald[10,11] who proved under certain hypotheses (which are all satisfied by a solution of the VlasovEinstein system) that in type IX the mean curvature cannot remain negative for an infinite time. If it never becomes positive then, since it is non-decreasing, it must be identically zero for all $t$ greater than some $t_{0}$. However it follows from the equations that in this case the 3-dimensional metric must be flat, contradicting the fact that the Bianchi type is IX.

\section{Kantowski-Sachs models}

In terms of Gauss coordinates based on a hypersurface of homogeneity, the KantowskiSachs metrics are of the form:

$$
d s^{2}=-d t^{2}+a^{2} d x^{2}+b^{2}\left(d \theta^{2}+\sin ^{2} \theta d \phi^{2}\right)
$$

where the functions $a$ and $b$ depend only on $t$. They are spherically symmetric. Regular frames invariant under this symmetry do not exist and so an approach must be used which is different from that of the previous section. The Vlasov equation will be written in terms of the locally defined orthonormal frame:

$$
\partial / \partial t, a^{-1} \partial / \partial r, b^{-1} \partial / \partial \theta, b^{-1} \csc \theta \partial / \partial \phi
$$

When written in terms of this frame the Vlasov equation for general functions in a geometry of the form (3.1) involves coefficients which are not regular functions on spacetime. However for distribution functions which are spherically symmetric these disappear. In the case of distribution functions $f$ with full Kantowski-Sachs symmetry, i.e. spherically symmetric and independent of $x$, the equation takes the form:

$$
\partial f / \partial t-a^{-1} \dot{a} v^{1} \partial f / \partial v^{1}-b^{-1} \dot{b}\left(v^{2} \partial f / \partial v^{2}+v^{3} \partial f / \partial v^{3}\right)=0 .
$$

In an orthonormal frame the expressions for the components of the energy momentum tensor are standard.

$$
\begin{aligned}
& \rho=\int f\left(1+|v|^{2}\right)^{1 / 2} d v^{1} d v^{2} d v^{3}, \\
& p_{i}=\int f\left(v^{i}\right)^{2}\left(1+|v|^{2}\right)^{-1 / 2} d v^{1} d v^{2} d v^{3} .
\end{aligned}
$$

Here $\rho$ is got by contracting $T_{\alpha \beta}$ twice with $e_{0}$ and $p_{i}$ is got by contracting it twice with $e_{i}$. Equations (2.1)-(2.4) apply to the present situation provided the indices are now interpreted as coordinate indices. A local existence theorem for solutions of the Vlasov-Einstein system with Kantowski-Sachs symmetry can now be proved by following 
the pattern of the proof of Lemma 2.1. The arguments leading up to Lemma 2.2 are also applicable in the present case. In fact the arguments are more transparent since the metric and second fundamental form are diagonal. The result of all this is the following:

Lemma 3.1 Let $\left(g^{0}, k^{0}, f^{0}\right)$ be an initial data set for equations (2.3), (2.4) and (2.8) which has Kantowski-Sachs symmetry, satisfies the constraints (2.1) and (2.2) and is such that $f^{0}(v)$ is a $C^{1}$ function of compact support. Then there exists a unique corresponding maximal $C^{1}$ solution $(g, k, f)$ on an interval $\left[0, T^{*}\right)$. If $H$ is bounded on $\left[0, T^{*}\right)$ then $T^{*}=\infty$.

In order to determine the global behaviour of $H$ it is enough, as in the Bianchi case, to find out whether in a solution where the function $H$ is initially negative it will eventually be positive. An affirmative answer to this question has been obtained by Burnett[2]. As before the equation (2.35) then implies that $H$ goes to infinity in finite time. Thus all Kantowski-Sachs solutions of the Vlasov-Einstein system exist only for a finite time in Gauss coordinates and the mean curvature of the hypersurfaces of homogeneity in such a solution takes on each real value precisely once.

\section{Curvature singularities}

For any (not necessarily spatially homogeneous) solution of the Vlasov-Einstein system the particle current density is defined by

$$
N^{\alpha}=-\int f p^{\alpha}\left|\operatorname{det}^{(4)} g\right|^{1 / 2} / p_{0} d p^{1} d p^{2} d p^{3},
$$

where $p^{\alpha}$ denotes the coordinate components of the momentum. It satisfies

$$
\nabla_{\alpha} N^{\alpha}=0 .
$$

Now suppose that some region of spacetime is foliated by compact spacelike hypersurfaces $S_{t}$ with normal vector $t^{\alpha}$. Then the quantity $\int_{S_{t}} N^{\alpha} t_{\alpha} d V_{g}$, where $d V_{g}$ is the volume element of the induced metric on the hypersurface, is time independent. In the case of a homogeneous spacetime it seems reasonable to hope that the integral can be dispensed with. For a Bianchi model $N^{0}$ takes the form:

$$
N^{0}=\int f(\operatorname{det} g)^{1 / 2} d v^{1} d v^{2} d v^{3} .
$$

Using this equation together with (2.8) and (2.12), the following identity can be derived:

$$
\partial_{t}\left((\operatorname{det} g)^{1 / 2} N^{0}\right)=-C_{i j}^{i} g^{j k} T_{0 k}(\operatorname{det} g)^{1 / 2} .
$$

In the Class A models where, by definition, $C_{i j}^{i}=0$ the right hand side of (4.4) vanishes and $(\operatorname{det} g)^{1 / 2} N^{0}$ is independent of time. For a Kantowski-Sachs spacetime (4.3) is replaced by

$$
N^{0}=\int f d v^{1} d v^{2} d v^{3}
$$


and a short computation shows that once more $(\operatorname{det} g)^{1 / 2} N^{0}$ is constant in time.

Now consider a solution of the Vlasov-Einstein system of the type discussed in the previous two sections and suppose that for this solution $H$ tends to infinity in finite time. Let $T^{*}$ be the time when $H$ becomes infinite. The proof which was used to show that this happens in those cases was a comparison with an explicitly solvable ordinary differential equation. In fact more information can be extracted from this method. It can be seen that an inequality of the form $H(t) \geq C\left(T^{*}-t\right)^{-1}$ holds. This inequality implies that det $g$ tends to zero as the singularity is approached. Hence for Kantowski-Sachs and Class A Bianchi models $N^{0}$ tends to infinity. Comparing the definitions shows that $T^{00} \geq N^{0}$ and so in these cases $T^{00}$ also diverges as the singularity is approached. Furthermore the scalar $T^{\alpha \beta} T_{\alpha \beta}$ is greater than or equal to $\left(T^{00}\right)^{2}$. The Einstein equations then imply that the curvature invariant $G^{\alpha \beta} G_{\alpha \beta}$ diverges as the singularity is approached. By replacing $t$ by $-t$ this result can also be applied to what is, with the convention for the time direction chosen in section 2 , the initial singularity. Thus the following result has been proved:

Theorem 4.1 Let $(g, k, f)$ be a non-vacuum locally spatially homogeneous solution of the Vlasov-Einstein system with Kantowski-Sachs or Bianchi Class A symmetry which is the maximal Cauchy development of data on a homogeneous hypersurface. Then every inextendible past-directed causal geodesic runs into a curvature singularity. If the symmetry type is Kantowski-Sachs or Bianchi IX then in addition every inextendible future-directed causal geodesic runs into a curvature singularity.

This theorem shows that for Kantowski-Sachs and Bianchi IX models the maximal Cauchy development of any non-vacuum initial datum for the Vlasov-Einstein system is inextendible. Since non-vacuum initial data constitute a generic subset of all initial data for the Vlasov-Einstein system for any reasonable definition of genericity, this suffices to prove strong cosmic censorship within these two symmetry classes. It is, however, still interesting to ask what happens in the vacuum case. (When investigating cosmic censorship in inhomogeneous cosmological models it will be necessary to consider cases where there is matter somewhere and vacuum elsewhere.) The only vacuum Kantowski-Sachs model with a compact Cauchy hypersurface is obtained by identifying the part of the Schwarzschild solution inside the event horizon by means of the transformation $t \mapsto t+C$. Here $t$ is the standard Schwarzschild time coordinate and $C$ a positive constant. This spacetime has a curvature singularity in the future but in the past the Schwarzschild event horizon is now a Cauchy horizon, through which the spacetime can be extended to a region with closed timelike curves. Thus the vacuum solution behaves very differently from the solutions containing matter. In the Bianchi IX case it does not seem to be known in general which vacuum initial data have maximal Cauchy developments which are extendible. However there is an example where the spacetime is extendible in this way both in the past and the future, namely the Taub-NUT solution. Siklos[22] has shown that the Taub-NUT solution is the only only Bianchi type IX vacuum solution which can be extended to a homogeneous spacetime containing a Cauchy horizon and so it is tempting to conjecture that it is the only one which is extendible beyond the maximal Cauchy development of data on a homogeneous spacelike hypersurface. 


\section{Geodesic completeness}

To investigate the geodesic completeness of homogeneous spacetimes which expand indefinitely, consider first the following consequence of (2.27) and the Hamiltonian constraint.

$$
\partial_{t} H=\frac{1}{4}\left(R+H^{2}+3 k^{i j} k_{i j}\right)+4 \pi g^{i j} T_{i j} .
$$

This section is concerned with spacetimes of Bianchi type I. In these spacetimes $R=0$ and so (5.1) implies the inequality

$$
\partial_{t} H \geq \frac{1}{2} H^{2},
$$

thus improving on (2.35). Comparing the inequality (5.2) with the corresponding differential equation gives:

$$
H(t) \geq-2(t+C)^{-1},
$$

for some constant C. Hence it can be concluded from (2.12) that

$$
\operatorname{det} g \leq C t^{4} .
$$

It can also be concluded, using the Hamiltonian constraint, that

$$
T_{00} \leq(1 / 4 \pi)(t+C)^{-2} .
$$

A special feature of Bianchi I spacetimes is that the Vlasov equation can be solved explicitly. Why this is so and why the same technique cannot be used for other Bianchi types is explained in [12]. For Bianchi type I the characteristic equation (2.11) implies that $d V_{i} / d s=0$. Thus if the distribution function is expressed in terms of $v_{i}$ rather than $v^{i}$ the Vlasov equation just says that it is independent of time. Hence

$$
T_{00}(t)=\int f\left(v_{i}\right)\left(1+g^{r s}(t) v_{r} v_{s}\right)^{1 / 2}(\operatorname{det} g(t))^{-1 / 2} d v_{1} d v_{2} d v_{3} .
$$

Note that in this equation $f$ does not depend on $t$. The matrix $g^{i j}(t)$ is symmetric and so there must exist a basis which is orthonormal with repect to $\delta_{i j}$ and consists of eigenvectors of this matrix. At any time there must be at least one of these which corresponds to the largest eigenvalue. Call it $w_{i}$. Then in the matrix notation introduced in section 2 $g^{i j} w_{i} w_{j}=\left\|g^{-1}\right\|$. Moreover

$$
g^{i j} v_{i} v_{j} \geq g^{r s} w_{r} w_{s}\left(\delta^{i j} v_{i} w_{j}\right)^{2} .
$$

Assume that $f$ is not identically zero and consider the expression

$$
F\left(y_{i}\right)=\int f\left(v_{i}\right)\left(\delta^{i j} v_{i} y_{j}\right) d v_{1} d v_{2} d v_{3} .
$$

If $y_{i}$ is any vector which lies on the unit sphere in the sense that $\delta^{i j} y_{i} y_{j}=1$ then clearly $F\left(y_{i}\right)$ is a positive quantity which depends continuously on $y_{i}$. Hence it must have a 
positive minimum $m$ on the sphere and $m$ depends only on $f$. Putting these facts together leads to the inequality

$$
T_{00}(t) \geq m\left\|g^{-1}\right\|^{1 / 2}(\operatorname{det} g)^{-1 / 2}
$$

Using (5.4) and (5.5) in this shows that $\left\|g^{-1}\right\|$ is bounded. Hence each component $g^{i j}$ is bounded and $g^{i j} V_{i} V_{j}$ is bounded along any causal geodesic.

It is obvious that when a homogeneous spacetime described in Gauss coordinates exists for all $t \geq 0$ then $t$ must tend to infinity along any inextendible future-directed causal geodesic. Geodesic completeness can therefore be decided by looking at the relation between $t$ and an affine parameter along such a geodesic. They are related by

$$
d \tau / d t=\left(l^{2}+g^{i j} V_{i} V_{j}\right)^{-1 / 2}
$$

where $l$ is the length of the tangent vector $t^{\alpha}$ to the geodesic, i.e. $\left(-t^{\alpha} t_{\alpha}\right)^{1 / 2}$. Since $g^{i j} V_{i} V_{j}$ is bounded it follows that the right hand side of (5.10) can be bounded from below by a positive constant. Hence when (5.10) is integrated to get $\tau$ the integral diverges as $t \rightarrow \infty$ and this is exactly what is needed to show future geodesic completeness.

Theorem 5.1 Let $(g, k, f)$ be a non-vacuum locally spatially homogeneous solution of the Vlasov-Einstein system with Bianchi I symmetry which is the maximal Cauchy development of data on a spacelike hypersurface. Then every inextendible future-directed causal geodesic is defined for arbitrarily large positive values of the affine parameter.

Together with the results of section 4 this proves strong cosmic censorship in the class of Bianchi I solutions of the Vlasov-Einstein system. Once again it is interesting to ask what happens in the vacuum case. The vacuum equations for Bianchi I spacetimes can be solved explicitly to obtain the Kasner solutions[23]. It is straightforward to check that these spacetimes are future geodesically complete and that except in one special case (which is just a piece of Minkowski space in disguise) there is a curvature singularity in the past where the curvature invariant $R^{\alpha \beta \gamma \delta} R_{\alpha \beta \gamma \delta}$ blows up. In the exceptional case there is a Cauchy horizon in the past. When the spatial topology is that of a torus the spacetime can be extended to a region containing closed timelike curves. The general vacuum solution has a very different behaviour at large times from the case where matter is present. It becomes more and more anisotropic as $t \rightarrow \infty$, contracting in one direction and expanding in two. The estimates for $g^{i j}$ obtained above show that when matter is present indefinite contraction in any one direction is impossible. In this sense the non-vacuum Bianchi type I solutions of the Vlasov-Einstein system resemble the Bianchi solutions with dust, which can be found explicitly[7].

Acknowledgements I thank the Syracuse relativity group for hospitality during the early stages of this work and in particular Jorma Louko for various discussions on Bianchi models.

\section{References}

1.A. ASHTEKAR AND J. SAMUEL, Class. Quantum Grav. 8 (1991), 2191.

2.G. A. BURNETT, Phys. Rev. D 43 (1991), 1143.

3.P. CHRUŚCIEL, Ann. Phys. (N.Y.) 202 (1990), 100. 
4.P. CHRUŚCIEL, J. ISENBERG AND V. MONCRIEF, Class. Quantum Grav. 7(1990), 1671.

5.D. EARDLEY AND V. MONCRIEF, Gen. Rel. Grav. 13 (1981), 887.

6.Y. FUJIWARA, H. ISHIHARA AND H. KODAMA, Class. Quantum Grav. 10 (1993), 859.

7.O. HECKMANN AND E. SCHÜCKING, in 'Gravitation: an Introduction to Current Research' (L. Witten Ed.) Wiley, New York, 1962.

8.J. ISENBERG AND V. MONCRIEF, Ann. Phys. (N.Y.) 199 (1990), 84.

9.R. T. JANTZEN in 'Cosmology of the Early Universe,' (L. Z. Fang and R. Ruffini Eds.) World Scientific, Singapore, 1984. 10.X.-F. LIN AND R. WALD, Phys. Rev. D 40 (1989), 3280.

11.X.-F. LIN AND R. WALD, Phys. Rev. D 41 (1990), 2444.

12.R. MAARTENS AND S. D. MAHARAJ, Gen. Rel. Grav. 22 (1990), 595.

13.V. MONCRIEF, Ann. Phys. (N.Y.) 132 (1981), 87.

14.G. REIN, Static solutions of the spherically symmetric Vlasov-Einstein system, Preprint gr-qc/9304028 (1993).

15.G. REIN AND A. D. RENDALL Commun. Math. Phys. 150 (1992), 561.

16.G. REIN AND A. D. RENDALL Commun. Math. Phys. 150 (1992), 585.

17.G. REIN AND A. D. RENDALL, Smooth static solutions of the spherically symmetric Vlasov-Einstein system, Ann. Inst. H. Poincaré (Physique Théorique) (to appear).

18.G. REIN AND A. D. RENDALL, Global existence of classical solutions to the VlasovPoisson system in a three dimensional, cosmological setting, Preprint (1993).

19.G. REIN, A. D. RENDALL AND J. SCHAEFFER, A regularity theorem for solutions of the spherically symmetric Vlasov-Einstein system, Preprint (1993).

20.A. D. RENDALL in 'Approaches to Numerical Relativity,' (R. d'Inverno Ed.) Cambridge University Press, Cambridge, 1992.

21.A. D. RENDALL, The Newtonian limit for asymptotically flat solutions of the VlasovEinstein system, Preprint MPA 722, gr-qc/9303027 (1993).

22.S. T. C. SIKLOS, Commun. Math. Phys. 58 (1978), 255.

23.R. WALD, 'General Relativity,' University of Chicago Press, Chicago, 1984. 